\title{
Galaxy And Mass Assembly (GAMA): the mass-metallicity relationship
}

\author{
C. Foster ${ }^{1,2}$, A. M. Hopkins ${ }^{3}$, M. Gunawardhana ${ }^{4}$, M. A. Lara-López ${ }^{3}$, R. G. Sharp ${ }^{5}$, O. Steele ${ }^{6}$, E. N. Taylor ${ }^{4}$, \\ S. P. Driver ${ }^{7,8}$, I. K. Baldry ${ }^{9}$, S. P. Bamford ${ }^{10}$, J. Liske ${ }^{11}$, J. Loveday ${ }^{12}$, P. Norberg ${ }^{13}$, J. A. Peacock ${ }^{14}$, M. Alpaslan ${ }^{7,8}$, \\ A. E. Bauer ${ }^{3}$, J. Bland-Hawthorn ${ }^{4}$, S. Brough ${ }^{3}$, E. Cameron ${ }^{15}$, M. Colless ${ }^{3}$, C. J. Conselice ${ }^{10}$, S. M. Croom ${ }^{4}$, \\ C. S. Frenk ${ }^{13}$, D. T. Hill ${ }^{8}$, D. H. Jones ${ }^{16}$, L. S. Kelvin ${ }^{7,8}$, K. Kuijken ${ }^{17}$, R. C. Nichol ${ }^{6}$, M. S. Owers ${ }^{3}$, H. R. Parkinson ${ }^{14}$, \\ K. A. Pimbblet $^{16}$, C. C. Popescu ${ }^{18}$, M. Prescott ${ }^{9}$, A. S. G. Robotham ${ }^{7,8}$, A. R. Lopez-Sanchez ${ }^{3,19}$, W. J. Sutherland ${ }^{20}$, \\ D. Thomas ${ }^{6,21}$, R. J. Tuffs ${ }^{22}$, E. van Kampen ${ }^{11}$, and D. Wijesinghe ${ }^{4}$
}

(Affiliations can be found after the references)

Received 18 July 2012 / Accepted 7 September 2012

\begin{abstract}
Context. The mass-metallicity relationship (MMR) of star-forming galaxies is well-established, however there is still some disagreement with respect to its exact shape and its possible dependence on other observables.

Aims. We measure the MMR in the Galaxy And Mass Assembly (GAMA) survey. We compare our measured MMR to that measured in the Sloan Digital Sky Survey (SDSS) and study the dependence of the MMR on various selection criteria to identify potential causes for disparities seen in the literature.

Methods. We use strong emission line ratio diagnostics to derive oxygen abundances. We then apply a range of selection criteria for the minimum signal-to-noise in various emission lines, as well as the apparent and absolute magnitude to study variations in the inferred MMR.

Results. The shape and position of the MMR can differ significantly depending on the metallicity calibration and selection used. After selecting a robust metallicity calibration amongst those tested, we find that the mass-metallicity relation for redshifts $0.061 \lesssim z \lesssim 0.35$ in GAMA is in reasonable agreement with that found in the SDSS despite the difference in the luminosity range probed.

Conclusions. In view of the significant variations of the MMR brought about by reasonable changes in the sample selection criteria and method, we recommend that care be taken when comparing the MMR from different surveys and studies directly. We also conclude that there could be a modest level of evolution over $0.06 \leq z \leq 0.35$ within the GAMA sample.
\end{abstract}

Key words. galaxies: abundances - galaxies: fundamental parameters - galaxies: star formation - galaxies: statistics

\section{Introduction}

The mass-metallicity relationship (MMR) describing a correlation between stellar mass and gas-phase metallicity in galaxies was first reported by Lequeux et al. (1979). Looking at spectra of $\mathrm{H}$ II regions for a small sample of irregular and blue compact galaxies, they found that metallicity and stellar mass were correlated over two orders of magnitudes in mass. Subsequently, with the advent of large spectroscopic surveys of galaxies, the reality of the MMR has been confirmed and established many times since, for both stellar (e.g., Gallazzi et al. 2005; Mendel et al. 2009) and gas-phase (e.g., Tremonti et al. 2004; Zahid et al. 2011) metallicities.

Several mechanisms have been proposed to explain the origins of the MMR. These include outflows, with enriched gas being retained preferentially by high-mass galaxies due to the increased depth of their potential well (e.g., Larson 1974; Kobayashi et al. 2007; Spitoni et al. 2010). Another possible mechanism is the interplay between the outflow of enriched gas and infall of pristine gas from the inter-galactic medium (e.g., Finlator \& Davé 2008). The downsizing phenomenon, wherein stars found in higher-mass galaxies today formed rapidly at earlier epochs suggests the surrounding gas should be enriched early. Stars found in low mass, low redshift galaxies, however, have lower $\alpha$-element abundance ratios, suggesting they formed slowly over longer periods (e.g., Cowie et al. 1996; Kodama et al. 2004; Abraham et al. 2005; Thomas et al. 2010). Indeed,
Garnett (2002) suggest that the MMR can be explained by the fact that low mass galaxies have higher gas fractions than high mass ones, implying that they are still converting gas into stars. Moreover, Rodrigues et al. (2012) show that galaxies at redshift $z \sim 2.2$ have higher gas fractions than their local counterparts, suggesting that evolution of the MMR should be expected under this scenario. A fourth possible mechanism is a stellar mass dependent or star formation rate (SFR) dependent evolving initial mass function (IMF), which has also been proposed as a viable scenario (e.g., Köppen et al. 2007; Wilkins et al. 2008a,b; Spitoni et al. 2010; Gunawardhana et al. 2011; Ferreras et al. 2012). This would also impact the rate and level of metallicity enrichment with galaxy stellar mass.

The Sloan Digital Sky Survey (SDSS) has allowed for the unequivocal confirmation of the MMR (Tremonti et al. 2004, hereafter T04). The method used in T04 is based on simultaneous fitting to all available emission lines using stellar population models. While using all the available lines to make a best estimate, this approach is more sensitive to noise. This method is ultimately tied to the reliability of the models used, and artificially produces quantised output metallicities as finite values of metallicities are modeled. T04 provide a conversion from the popular $R 23=\left(\left[\mathrm{O}_{\mathrm{II}}\right] \lambda 3727+[\mathrm{O}\right.$ III $\left.] \lambda \lambda 4959,5007\right) / \mathrm{H} \beta$ strong emission line ratio to their calibration.

Kewley \& Ellison (2008, hereafter KE08) have used the SDSS data to compare the inferred metallicities using 
various calibrations. Empirical conversions between the different metallicity calibrations are provided. They find that the absolute position and shape of the MMR can vary significantly from one calibration to another, emphasising the need to use similar metallicity calibrations when comparing different samples. Indeed, KE08 and other authors (e.g. López-Sánchez \& Esteban 2010; Bresolin et al. 2009; Moustakas et al. 2010) have shown that metallicities derived using calibrations based on photoionization models (e.g., McGaugh 1991; KD02; KK04) tend to be systematically $0.2-0.4$ dex higher than metallicities derived using the direct method (i.e., using a direct estimation of the electron temperature) or calibrations based on it (e.g., PP04; Pilyugin \& Thuan 2005). In addition, depending on the metallicity calibration used, KE08 found that the turnover of the MMR depends on the aperture covering fraction. To avoid such effects, they recommend a minimum redshift of $z=0.04$ for the SDSS $3^{\prime \prime}$ aperture fibre spectra. Similarly, Moustakas et al. (2011) find a measurable dependence of the MMR on the metallicity calibration used and aperture biases using a sample of 3000 galaxies from the AGN and Galaxy Evolution Survey (AGES). They also find that the MMR is affected by contamination from Active Galactic Nuclei (AGN). Hence, differences induced by metallicity calibrations, aperture effects and AGN contamination need to be taken into account when comparing results from different surveys and studies.

Several groups have studied the redshift evolution of the MMR and sometimes find conflicting results, particularly at lower redshifts. Both Lara-López et al. (2009a,b) and Savaglio et al. (2005) detect evolution of the MMR up to $z=0.4$ using SDSS, and over $0.4 \leq z \leq 1.0$ from a combined sample from the Gemini Deep Deep Survey (GDDS) and the Canada-France Redshift Survey (CFRS), respectively. In sharp contrast, Carollo \& Lilly (2001) do not find significant evolution when comparing the MMR in a sample of galaxies at $0.5 \leq z \leq 0.7$ from CFRS to that locally observed. Yet, several groups independently detect significant evolution at comparable redshifts $0.4 \lesssim z \lesssim 1.0$ (see e.g., Kobulnicky \& Kewley 2004; Mouhcine et al. 2006; Rodrigues et al. 2008; Morelli et al. 2012). Pushing the redshift barrier further, Erb et al. (2006) analyse a sample of 87 UV selected star forming galaxies to show that galaxies at high redshift $(z \sim 2)$ not only have lower oxygen abundances, but also higher gas fraction ( $\sim 50$ per cent). At redshifts $z>3$, Maiolino et al. (2008) select a variety of independent ratios and calibrations to obtain metallicities and detect an evolution of the MMR and its slope in a sample of 9 star forming galaxies observed with ESO-VLT. They claim that no hierarchical formation simulation reproduces the MMR at $z \sim 3$, while monolithic collapse simulations do. At even higher redshift, Laskar et al. (2011) obtain a sample of 20 gamma ray bursts at redshifts $3 \leq z \leq 5$ and demonstrate that the MMR at high redshift is significantly offset to lower metallicities than that at $z \lesssim 3$.

Another fundamental observable parameter that has proven to be important in order to disentangle which scenario(s) is (are) responsible for the MMR is the SFR. Here again, there are some discrepancies in the various findings about the dependence of the MMR on SFR. Ellison et al. (2008) select galaxies in the SDSS to study the dependence of the MMR on the specific SFR (i.e., SFR per unit mass, hereafter SSFR). They observe that galaxies with higher SSFR for their stellar mass have lower metallicities $(\log (\mathrm{O} / \mathrm{H})$ is as much as 0.2 dex lower). More recently, Mannucci et al. (2010, hereafter M10) have also used SDSS galaxies to study the inter-dependence between mass, metallicity and SFR. They claim that there exists a "fundamental relationship" or three-dimensional curved surface in stellar mass, SFR and metallicity space where all star forming galaxies lie. They also find that higher SFR galaxies (and lower metallicities) are selected at higher redshift, leading to an apparent evolution of the MMR. M10 demonstrate that high redshift galaxies from Erb et al. (2006), but not Mannucci et al. (2009) follow this "fundamental relationship". Similarly, Lara-López et al. (2010a) and Lara-López et al. (2010b, hereafter L10b) discovered a relationship which is best described as a "fundamental plane" between SFR, metallicity and stellar mass (recently updated by Lara-Lopez et al. 2012). In contrast to M10, they do not find any curvature in three-dimensions or any evolution of the fundamental plane up to $z \sim 3.5$, and detect a shallow but positive correlation between SFR and metallicity such that high SFR galaxies tend to have higher metallicities. In general, they observe that metallicities are lower, SFRs are higher and the morphology of galaxies shows a higher fraction of late-type galaxies at high redshift compared to locally. Using semi-analytic models, Yates et al. (2012) reproduce the 3-dimensional surface theoretically. They explain that low SFR, high mass galaxies have typically exhausted their gas reservoirs in a recent major merger, hence preventing further star formation. Subsequent inflow of metal-poor gas then dilutes the gas around these galaxies without significant further star formation.

The focus of this paper is to present the MMR using data from the Galaxy And Mass Assembly survey (GAMA, see Driver et al. 2011) ${ }^{1}$ and study its dependence on sample selection. A companion paper (Lara-López et al., in prep.) presents the joint distribution of GAMA and SDSS galaxies in the three-dimensional SFR/stellar mass/metallicity parameter space. GAMA is a good complement to the previous SDSS-based studies as it probes to fainter magnitudes than SDSS, in principle allowing for a range of lower stellar masses to be studied at a given redshift, and to higher redshift at a given stellar mass. The paper is structured as follows. The data and sample selection are described in Sect. 2. Sections 3 and 4 present our analysis and highlight our results, respectively. A summary and our conclusions are given in Sect. 5.

Throughout we assume a cosmology given by $\Omega_{\mathrm{M}}=0.3$, $\Omega_{\Lambda}=0.7$ and $H_{0}=70 \mathrm{~km} \mathrm{~s}^{-1} \mathrm{Mpc}^{-1}$.

\section{Data}

\subsection{GAMA survey}

Upon completion, the GAMA survey (see Driver et al. 2011) will include data from many telescopes (AAT, VST, VISTA, Herschel, ASKAP, and GALEX) to create a database ranging from radio to ultraviolet wavelengths for roughly 400000 galaxies over 360 square degrees. Optical spectra from AAOmega on the AAT have been obtained for $\sim 150000$ galaxies over three regions covering 144 square degrees in total as part of GAMA phase-I. The GAMA Phase-I spectroscopic completeness is $98 \%$ to an $r$-band Petrosian magnitude of 19.4 over two of the regions and $r \sim 19.8$ over the third region. GAMA Phase-II will include a larger volume and will be complete to an $r$-band Petrosian magnitude of 19.8. Because of its depth, it is a good complement to SDSS probing to lower stellar masses and higher redshift. This work uses GAMA Phase-I data.

We provide a brief summary of the spectroscopic processing, with full details provided by Hopkins et al. (in prep.). The blue and red parts of the AAOmega optical spectra are processed

\footnotetext{
${ }^{1}$ http://www.gama-survey.org/
} 


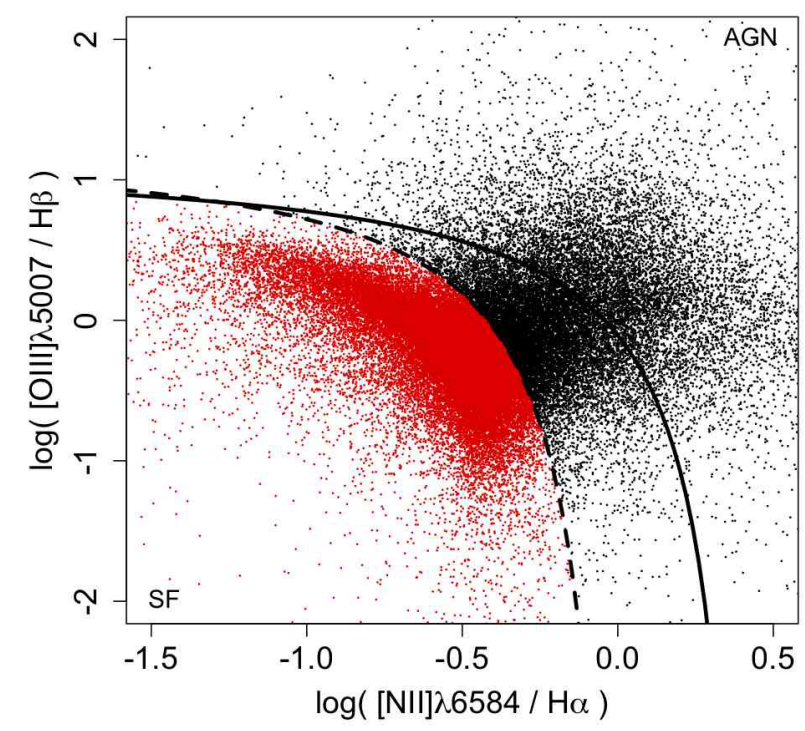

Fig. 1. Selection of star-forming galaxies (red) using the prescription from Kauffmann et al. (2003, dashed line) BPT diagram (Baldwin et al. 1981). The solid line shows the prescription by Kewley et al. (2001). Objects with AGN-like spectra are close to the upper right while starforming galaxies reside closer to the lower left corner as labelled.

and reduced separately using the 2DFDR data reduction pipeline (see Croom et al. 2004; Sharp \& Birchall 2010) before combination. The pipeline performs standard bias subtraction, flatfielding, tram-line fitting and wavelength calibration using arcs. Sky subtraction is done using dedicated sky fibres before performing optimal 1D extraction and is refined using Principal Component Analysis sky subtraction (Sharp \& Parkinson 2010). This is followed by flux calibration using standard star observations. Redshifts are derived from the 1D spectra following the process described in Driver et al. (2011).

\subsection{Emission line measurements}

We use the Gas AND Absorption Line Fitting algorithm (GANDAlf, Sarzi et al. 2006), an extension of the Penalized Pixel cross-correlation Fitting (PPXF) algorithm of Cappellari \& Emsellem (2004), to model the stellar absorption and emission line spectral components of our flux calibrated spectra. We measure the corrected flux and equivalent width of the strong emission lines from the GANDALF output emission line spectra. Our stellar population templates are from Maraston \& Strömbäck (2011) based on the code of Maraston (2005), which uses the MILES stellar library (Sánchez-Blázquez et al. 2006) with the revised spectral resolution Beifiori et al. (2011). Our emission line measurements are effectively stellar-absorption corrected through the template fitting performed by GANDALF. We use the Balmer decrement to apply the dust obscuration curve correction of Cardelli et al. (1989) to the emission line measurements, as recommended by Calzetti (2001).

\subsection{Sample selection}

We construct several subsamples of the GAMA data. First, we use the relationship from Kauffmann et al. (2003) to discriminate galaxies with significant AGN contribution from star-forming galaxies using their position in the BPT diagram (Baldwin et al. 1981). Figure 1 illustrates this selection. Galaxies whose $\mathrm{H} \alpha$
Table 1. Definition of the selected volume limited samples (Col. 1).

\begin{tabular}{lcccc}
\hline \hline $\begin{array}{l}\text { Sample } \\
(1)\end{array}$ & $\begin{array}{c}z_{\min } \\
(2)\end{array}$ & $\begin{array}{c}z_{\max } \\
(3)\end{array}$ & $\begin{array}{c}M_{\mathrm{r}, \max } \\
(4)\end{array}$ & $\begin{array}{c}M_{\mathrm{r}, \min } \\
(5)\end{array}$ \\
\hline 1 & 0.070 & 0.085 & -18.58 & -19.73 \\
2 & 0.085 & 0.100 & -18.97 & -20.18 \\
3 & 0.100 & 0.115 & -19.30 & -20.57 \\
4 & 0.115 & 0.130 & -19.60 & -20.90 \\
5 & 0.130 & 0.145 & -19.86 & -21.20 \\
6 & 0.145 & 0.160 & -20.10 & -21.46 \\
7 & 0.160 & 0.175 & -20.33 & -21.70 \\
8 & 0.175 & 0.190 & -20.53 & -21.93 \\
9 & 0.190 & 0.205 & -20.72 & -22.13 \\
10 & 0.205 & 0.235 & -21.07 & -22.32 \\
11 & 0.235 & 0.265 & -21.39 & -22.67 \\
12 & 0.265 & 0.295 & -21.67 & -22.99 \\
13 & 0.295 & 0.325 & -21.93 & -23.27 \\
14 & 0.325 & 0.350 & -22.13 & -23.53 \\
\hline
\end{tabular}

Notes. Redshift limits are given in Cols. 2 and 3, while absolute Petrosian magnitude limits can be found in Cols. 4 and 5 for each sample.

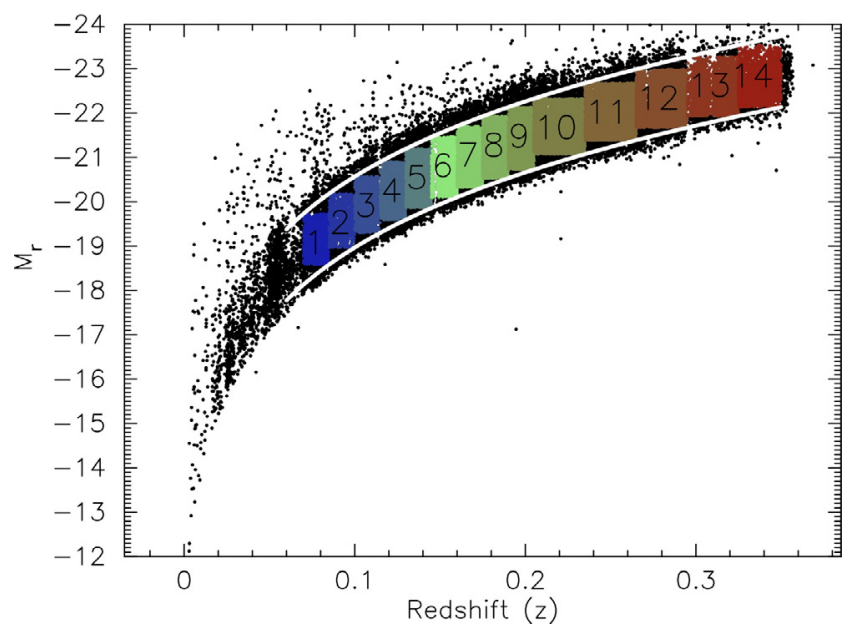

Fig. 2. The redshift and absolute $r$-band magnitude distribution of galaxies in our 14 GAMA volume limited samples (see Table 1). White lines show the lower and upper apparent magnitude completeness of GAMA.

or $\mathrm{H} \beta$ lines are affected by overlapping strong sky line residuals are rejected. We nominally select galaxies for which the strong emission lines $\mathrm{H} \alpha, \mathrm{H} \beta$ and $[\mathrm{NII}] \lambda 6583$ have $S / N \geq 3$. We refer to this selection as our "fiducial" selection throughout. A discussion of possible selection effects and justification of the fiducial selection are presented in Sect. 4.1. We reject any galaxies at $z<0.061$ to minimise fibre aperture issues as described in KE08 scaled for GAMA fibres (i.e., $2^{\prime \prime}$ in diameter). We also reject objects with a Balmer decrement $\mathrm{H} \alpha / \mathrm{H} \beta<2.5$ and extinction $E(B-V)>10$. We refer to this sample as the "main sample" for our analysis.

Finally, we also select many volume limited samples in narrow redshift ranges, with maximum and minimum absolute $r$-band Petrosian magnitude determined based on the survey apparent magnitude completeness limits (i.e., $17.8 \leq r_{\text {petro }} \leq 19.4$ ). This is done in order to have the broadest range in absolute magnitudes possible, yielding a maximal stellar mass range in each bin. The redshift ranges for each bin are given in Table 1 and shown in Fig. 2. 


\section{Analysis}

\subsection{Star formation rates}

We compute the SFR from the stellar absorption and extinction corrected $\mathrm{H} \alpha$ flux measured by GANDALF on the flux calibrated spectra using Eq. (2) of Kennicutt (1998). Because they are measured from the flux calibrated spectra, our SFR measurements include an implicit correction for fibre aperture effects. The implicit assumption made is that star formation is distributed across the galaxy following the stellar light (Hopkins et al. 2003). Throughout this work, we assume a Salpeter IMF (Salpeter 1955). Our SFR measurements compare well with those published in Gunawardhana et al. (2011) and SDSSmeasured values in Brinchmann et al. (2004). Throughout, SFR is quoted in units of $M_{\odot} \mathrm{yr}^{-1}$.

\subsection{Stellar masses}

Stellar mass measurements are described in Taylor et al. (2011). They are determined using spectral energy distribution fitting of the $u-, g-, r$-, $i$ - and $z$-magnitudes. We use a library of $\sim 80000$ templates from the Bruzual \& Charlot (2003) stellar population models. The dust curve of Calzetti (2001) is assumed during this process. The quoted stellar mass (in units of $M_{\odot}$ ) is derived from marginalisation over the posterior probability distribution of all fitted parameters (e.g., age, metallicity, stellar mass, star formation history, etc.). An offset of 0.2 is applied to the log of the stellar mass values published in Taylor et al. (2011) to accommodate our assumption of a Salpeter IMF.

\subsection{Gas-phase metallicities}

As stated in Sect. 1, several methods may be used to derive gas-phase metallicities of star-forming galaxies (see reviews by López-Sánchez \& Esteban 2010; Lopez-Sanchez et al. 2012). Although the direct method should be preferred, the faintness of the auroral lines (particularly, the [OIII] $\lambda 4363$ emission line) often prevents its use for relatively high metallicities $(12+\log (\mathrm{O} / \mathrm{H}) \geq 8.4)$. Hence, techniques using the more readily measurable strong emission-lines have been developed to estimate galaxy gas-phase metallicity. In this work, we present a representative set of three of the popular strong emission line methods discussed in the literature. These include techniques based on photoionization models (M91; KD02; KK04) and empirical calibrations from samples of objects with known metallicity (PP04).

\section{Method 1: Kewley \& Dopita (2002)}

First, we use the method described in KD02 with the update described in Appendix A of KE08. This can be summarised as a combination of the $N 2 O 2=[\mathrm{N} \mathrm{II}] \lambda 6583 /\left[\mathrm{O}_{\mathrm{II}}\right] \lambda 3727$ ratio and an average of several calibrations of the degenerate $R 23$ line ratio. For $\mathrm{N} 2 \mathrm{O} 2>-1.2$ we solve the following polynomial:

$$
\begin{aligned}
N 2 O 2= & 1106.8660-532.15451 Z+96.373260 Z^{2} \\
& -7.8106123 Z^{3}+0.23928247 Z^{4},
\end{aligned}
$$

where $Z=\log (\mathrm{O} / \mathrm{H})+12$.

If $\mathrm{N} 2 \mathrm{O} 2<-1.2$, we use the average of the Kobulnicky \& Kewley (2004, hereafter KK04) and McGaugh (1991, hereafter M91) methods. In M91, oxygen abundances $\left(Z_{M 91}\right)$ for the lower R23 branch are derived using:

$$
\begin{aligned}
Z_{\mathrm{M} 91}= & 12-4.944+0.767 x+0.602 x^{2}-y_{1} \\
& \times\left(0.29+0.332 x-0.331 x^{2}\right),
\end{aligned}
$$

where $y_{1}=\log \left([\mathrm{O} \mathrm{III}] \lambda \lambda 4959,5007 /\left[\mathrm{O}_{\mathrm{II}}\right] \lambda 3727\right)$ and $x=$ $\log R 23$. KK04 obtain oxygen abundances $\left(Z_{\mathrm{KK} 04}\right)$ by iterating the following two equations:

$$
\begin{aligned}
\log q= & \left(32.81-1.153 y_{2}^{2}+Z\left(-3.396-0.025 y_{2}+0.1444 y_{2}^{2}\right)\right) \\
& \times\left(4.603-0.3119 y_{2}-0.163 y_{2}^{2}+Z\right. \\
& \left.\left.-0.48+0.0271 y_{2}+0.02037 y_{2}^{2}\right)\right)^{-1}
\end{aligned}
$$

where $y_{2}=\log \left([\mathrm{O}\right.$ III $\left.] \lambda 5007 /\left[\mathrm{O}_{\mathrm{II}}\right] \lambda 3727\right)$ and

$$
Z_{\mathrm{KK} 04}=9.40+4.65 x-3.17 x^{2}-\log q\left(0.272+0.547 x-0.513 x^{2}\right),
$$

with $q$ the ionization factor, until convergence is achieved. Hence, for $N 2 O 2<-1.2$, the final quoted oxygen abundance is simply $12+\log (\mathrm{O} / \mathrm{H})=\left(Z_{\mathrm{M} 91}+Z_{\mathrm{KK} 04}\right) / 2$.

\section{Method 2: Pettini \& Pagel (2004)}

The second method used is that described in Pettini \& Pagel (2004, hereafter PP04). Briefly, we use the O3N2 ratio, which is defined as follows:

$O 3 N 2=\log \left(\frac{[\mathrm{OIII}] \lambda 5006 / \mathrm{H} \beta}{[\mathrm{NII}] \lambda 6583 / \mathrm{H} \alpha}\right)$.

In $\mathrm{PP} 04$, the $\mathrm{O} 3 \mathrm{~N} 2$ ratio abundances are calibrated using HII regions yielding the following relationship:

$12+\log (\mathrm{O} / \mathrm{H})_{P P 04}=8.73-0.32 \times 03 N 2$.

Because metallicities obtained directly using individual HII regions result in lower metallicities (e.g. KD02), we use the public SDSS line measurement (Brinchmann et al. 2004) and oxygen abundances (T04) to derive the following empirical conversion between PP04 and T04 abundances (see Lara-López et al., in prep.):

$12+\log (\mathrm{O} / \mathrm{H})=0.103+1.021 \times\left(12+\log (\mathrm{O} / \mathrm{H})_{P P 04}\right)$.

Equation (7) "accommodates" the well known 0.2-0.4 dex offset expected between metallicities derived using the direct and model based methods (see Sect. 1).

\section{Method 3: Kobulnicky \& Kewley (2004)}

The third abundance determination method presented in this work is that of KK04 as updated by KE08. It is based on the popular R23 ratio and hence yields representative results of studies that use this ratio. The lower $\mathrm{R} 23$ branch conversion is given by iterating Eqs. (3) and (4) until convergence. For N2O2 $\geq-1.2$, metallicities are derived by iterating Eq. (3) and

$$
\begin{aligned}
12+\log (\mathrm{O} / \mathrm{H})_{K K 04}= & 9.72-0.777 x-0.951 x^{2}-0.072 x^{3} \\
& -0.811 x^{4}-\log (q)(0.0737-0.0713 x \\
& \left.-0.141 x^{2}+0.0373 x^{3}-0.058 x^{4}\right)
\end{aligned}
$$

until convergence. 


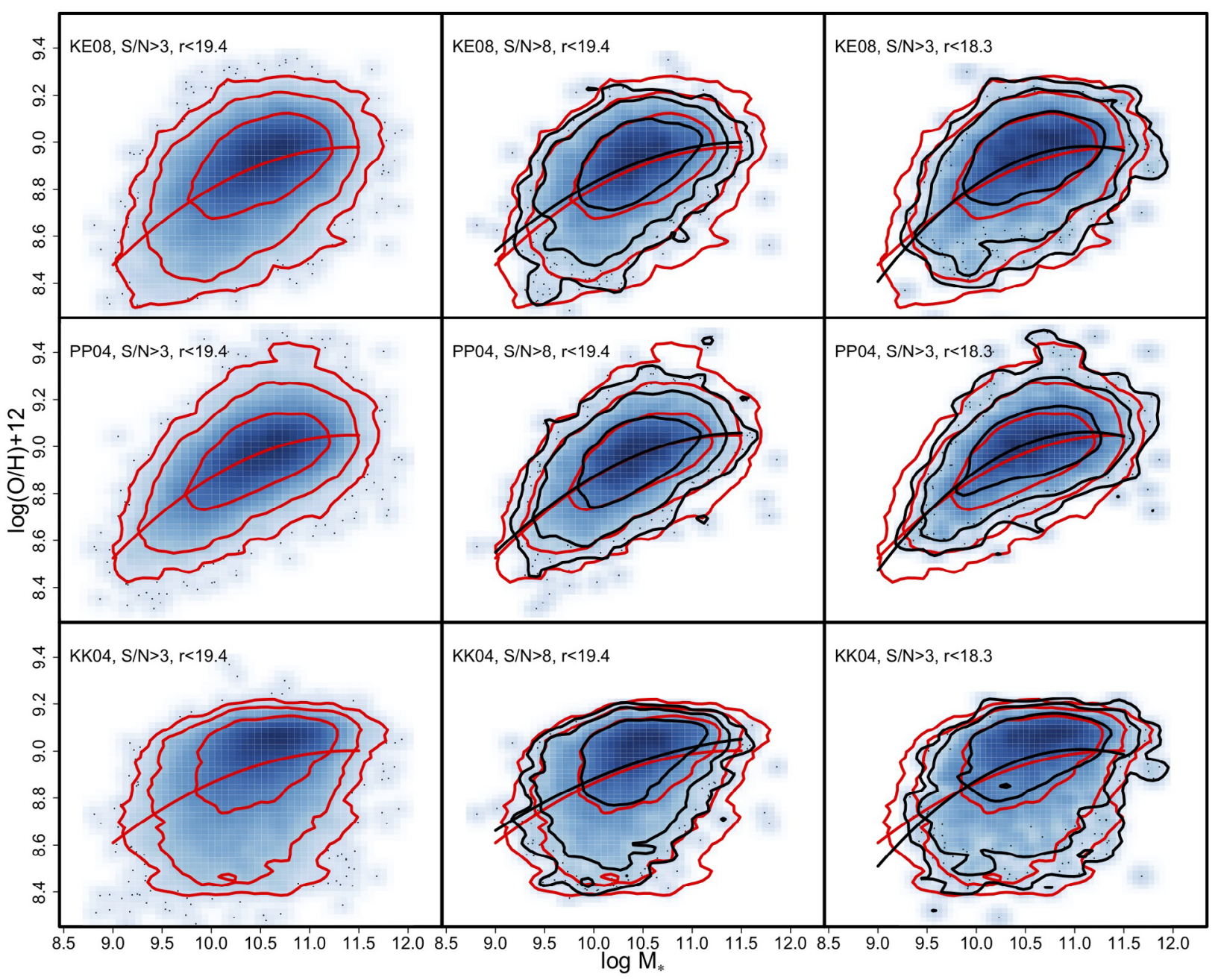

Fig. 3. A summary of the influence of varying the apparent selection criteria such as the survey apparent magnitude limit $(r)$ and $\mathrm{S} / \mathrm{N}$ limit in our fiducial lines $(\mathrm{H} \alpha, \mathrm{H} \beta$ and $[\mathrm{NII}] \lambda 6583$ ) for three representative metallicity calibrations as labelled: KE08 (top row), PP04 (middle row) and KK04 (lower row). For each row, red contours show the 1,2 and $3 \sigma$ profiles for our fiducial selection criteria, while the black contours show how varying $\mathrm{S} / \mathrm{N}$ ratio cut and survey apparent magnitude limit influence the position and shape of the MMR. The red and black solid lines are respective second order polynomial fits to the MMR data. The fitted position of the MMR varies slightly for all ratios, but these variations are small (i.e., typically $<0.05$ dex).

\section{Uncertainties}

In all cases, uncertainties are computed using Monte-Carlo methods. Assuming that the uncertainties on the measured emission line fluxes are normally distributed, we draw 1000 random measurements of the relevant emission lines for each object and recompute the metallicity each time. The quoted uncertainty for each calibration is the standard deviation of the relevant 1000 measurements.

For completeness, we also compute oxygen abundances using the methods described in KD02 for various available emission lines and the R23 calibrations of M91, Zaritsky et al. (1994) and T04. We choose to present the above mentioned methods of KE08, PP04 and the R23 calibration of KK04 as a representative selection of the various methods used in the literature. We find that these three methods summarize and represent well the range of results obtained from the variety of measurement methods.

\section{Results}

This section focuses on the sensitivity of the fitted MMR with a set of common apparent selection criteria and abundance calibrations. We also look for evidence of redshift evolution and dependency on SFR. The three-dimensional distribution of galaxy stellar mass, metallicity and SFR in the joint GAMA and SDSS samples is discussed in detail in a companion paper (Lara-López et al., in prep.).

\subsection{Mass-metallicity relation}

As mentioned in Sect. 2.3, we study the dependence of the MMR on selection criteria such as the signal-to-noise $(\mathrm{S} / \mathrm{N})$ in various lines and apparent magnitude completeness, as well as on the metallicity calibration used. For this, we compare the MMR obtained with our fiducial selection criteria with that obtained by increasing the $\mathrm{S} / \mathrm{N}$ limit from 3 to 8 , but keeping the $r$-band Petrosian magnitude limit constant. Next, we vary the $r$-band Petrosian magnitude limit between 19.4 and 18.3, while keeping the $\mathrm{S} / \mathrm{N}$ limit at 3 . In all cases, we fit a second order polynomial to the distribution obtained, following the approach of other recent work. In what follows, we consider vertical (metallicity) variations of order 0.05 dex to be significant, as they could account for at least half of the reported variations of the MMR with redshift for $z \lesssim 0.4$ (see for example Lara-López et al. 2009b) and specific SFR (e.g., Ellison et al. 2008). In other words, we are looking for vertical differences of either the contours or fitted MMR with variations of the selection. Figure 3 summarizes 


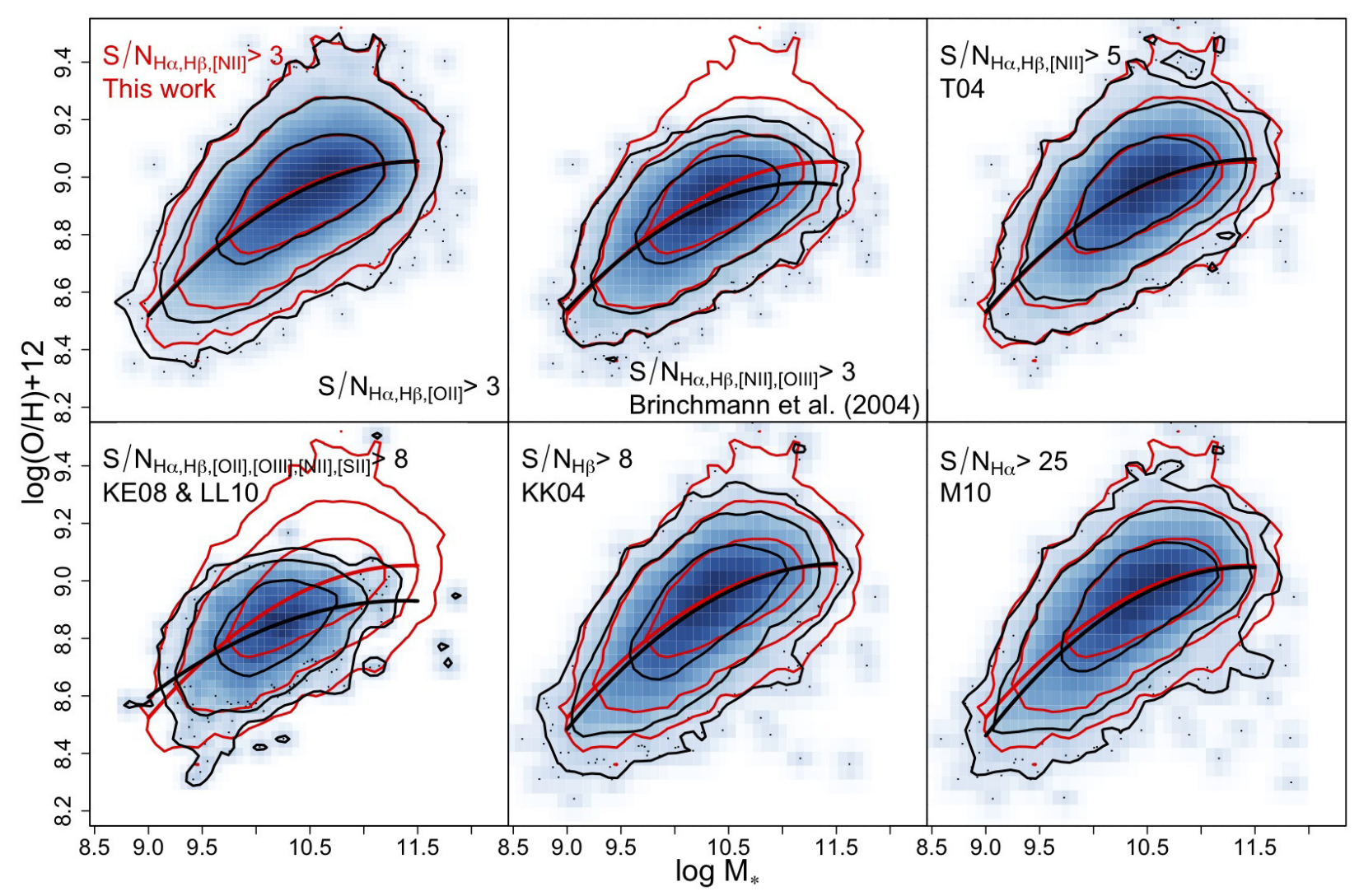

Fig. 4. GAMA MMR measured using the a variety of selection criteria taken mostly from the recent literature, as labeled, for the PP04 abundance estimate. The MMR obtained using the selection criteria of Brinchmann et al. (2004), T04, KE08, Lara-López et al. (2010a, LL10), Kobulnicky \& Kewley (2004, KK04) and Mannucci et al. (2010, M10) (respective black 1, 2 and $3 \sigma$ contours) are compared to that obtained using the selection criteria used in this work (red 1, 2 and $3 \sigma$ contours). The MMR measured varies significantly ( $>0.05$ dex) if one selects on the [OIII] $\lambda 5007$ line.

the results of this exercise. Varying these selection criteria for the MMR does not significantly alter the fitted MMR for a given calibration, however as emphasised by KE08, the shape and location of the MMR is significantly altered between the three calibrations shown.

Figure 4 shows that the MMR can be significantly different depending on the choice of selection criteria, for a variety of criteria that have been used in recent work. In general, we find that selecting on the [OIII] $\lambda 5007$ line most influences the position and shape of the measured MMR. This is a consequence of higher metallicities being associated with relatively fainter oxygen lines, which are more likely to show lower $\mathrm{S} / \mathrm{N}$. We note that the effect is more significant with the higher $\mathrm{S} / \mathrm{N}$ threshold applied by KE08 and LL10, compared to the lower threshold applied by Brinchmann et al. (2004), although even this cut introduces a measurable effect. We therefore recommend caution, and rigorously consistent sample selection if cuts are being made based on the $\mathrm{S} / \mathrm{N}$ ratio in this line, when studying small variations of the MMR.

An alternative way to identify galaxies with reliable metallicity measurements is to select based on the size of the metallicity uncertainty itself. The effects of such a selection are shown in Fig. 5, where we vary the selection on metallicity uncertainty from 0.1 to 0.01 dex. It can be seen that for reasonably moderate criteria, the MMR and contours are essentially unaltered, however applying an extreme cut does alter the shape and position of the MMR. We cannot reject the possibility that these could be due to genuine evolutionary effects since galaxies at lower redshift would inevitably have lower errors. More importantly, we insist that selecting based on overall metallicity uncertainties can only be applied for calibrations that are monotonic and smooth conversions between observables and metallicity. Piecewise calibrations such as the KD02 and R23-based methods have discontinuous conversion functions that yield large errors around the transition, hence a selection on error would discriminate against galaxies of those or similar metallicities (typically $12+\log (\mathrm{O} / \mathrm{H}) \sim 8.4 \mathrm{dex}$ ).

Figure 6 shows the MMR, the mass-SFR relation and the metallicity-SFR relation for GAMA. Each panel shows the relation for our main sample in greyscale, with the distribution for each of the 14 volume limited redshift samples overlayed as coloured contours. We emphasize that even though our volume limited samples are complete in a luminosity sense (absolute $r$-band magnitude), this does not correspond to a strict stellar mass limit. While each volume limited sample is itself complete, we observe the expected Malmquist-type bias as we consider samples selected at different redshift. Galaxies of low (high) stellar masses are preferentially observed in the lowest (highest) redshift samples. As a result, in each redshift bin, the range of stellar masses probed is limited. This complicates the study of possible evolution within GAMA. This limitation is addressed by combining the SDSS and GAMA samples by Lara-López et al. (in prep.).

Bearing all previously mentioned caveats in mind, we tentatively find that the MMR in GAMA is mostly consistent with that measured in SDSS. It is important to emphasize that even though GAMA metallicities are scaled to the same calibration as that used in the presented SDSS MMR they are not strictly measured using the same selection criteria and metallicity diagnostic. Hence, for the reasons already outlined above, its exact position 


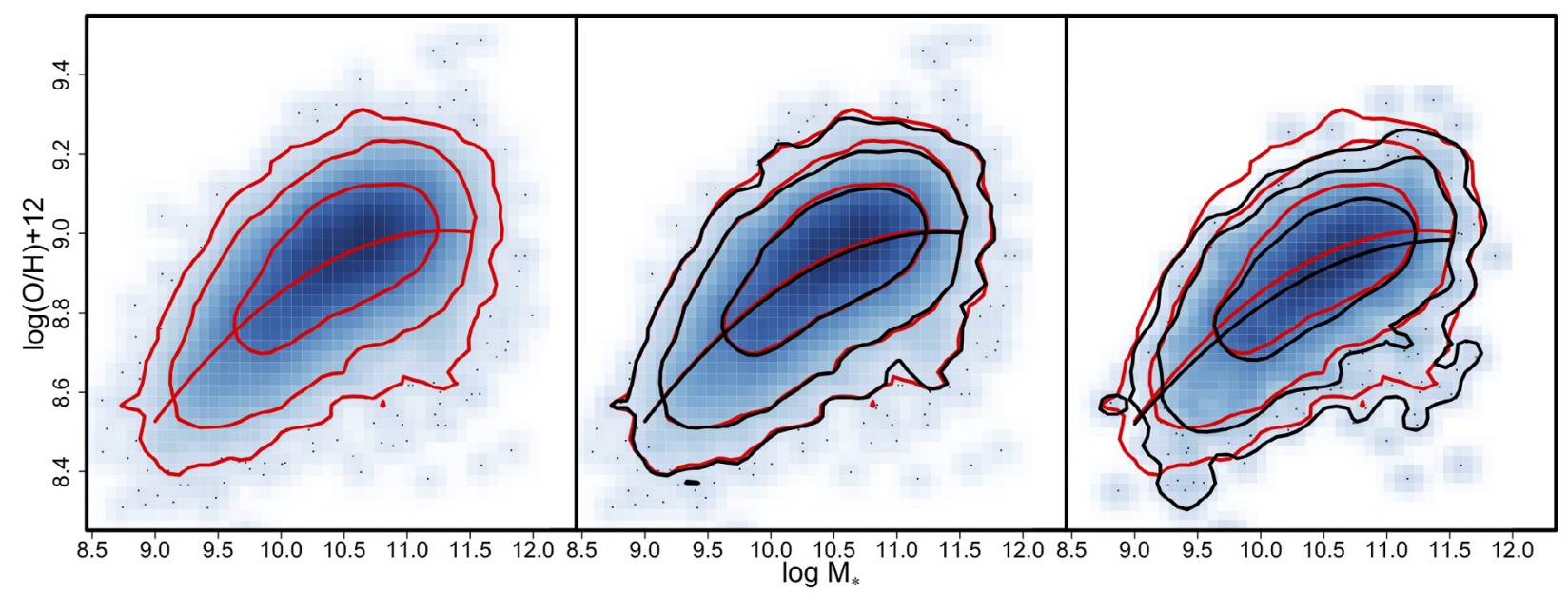

Fig. 5. GAMA MMR measured using galaxies selected with metallicity uncertainties less than 0.1 dex (left), 0.05 dex (middle) and 0.01 dex $($ right). Contours are similar to those of Figs. 3 and 4.
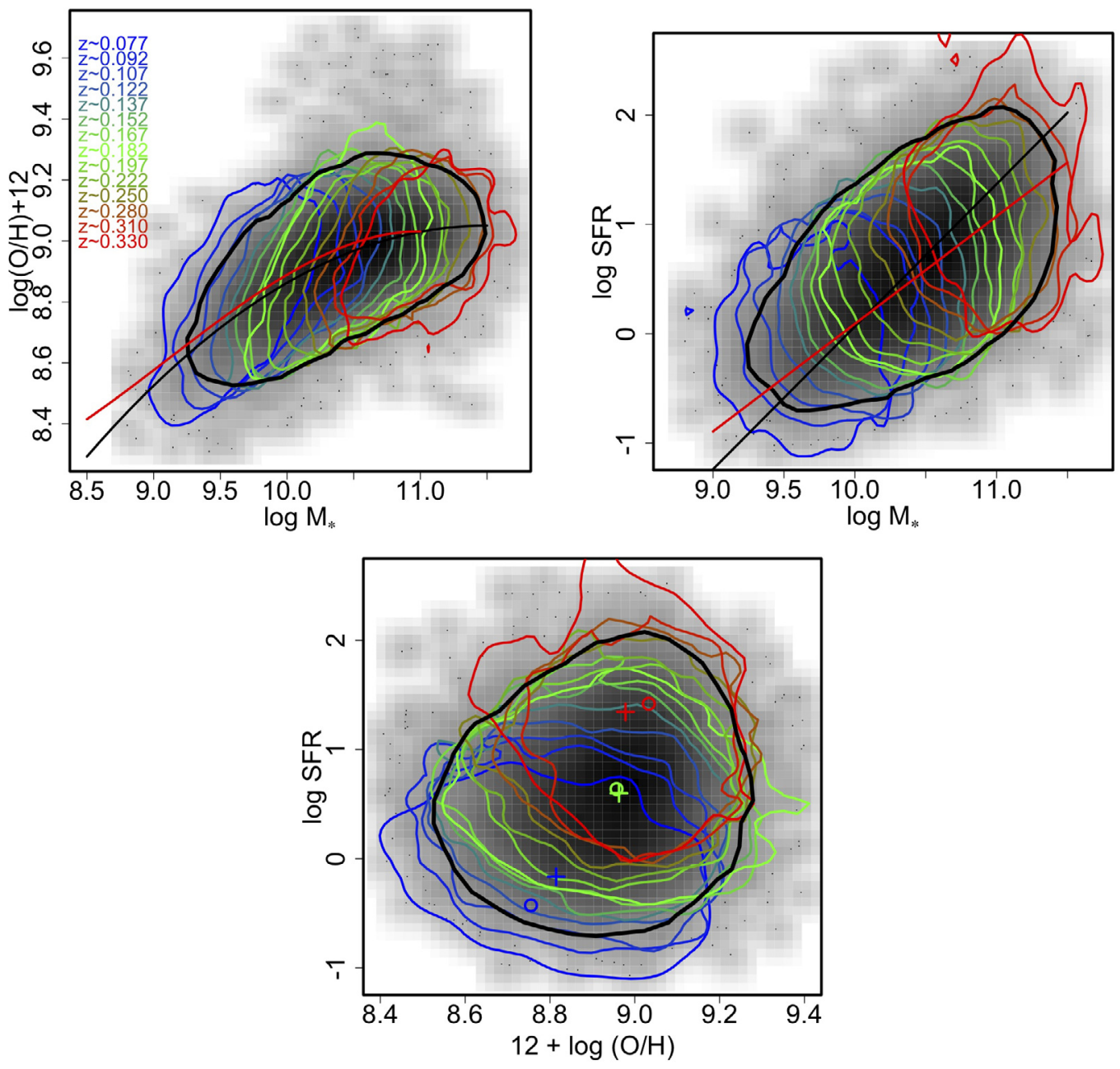

Fig. 6. The GAMA mass-metallicity (upper left), mass-SFR (upper right) and metallicity-SFR (lower-middle) relationships in our main sample. Black and coloured contours show $2 \sigma$ contours for the main and volume limited samples with median redshifts as labelled, respectively. Red and black solid lines show the fitted SDSS MMR from KE08 using the updated KD02 conversion and our fit to the GAMA data, respectively. Open circles in the lower panel show the expected mean metallicity and SFR as inferred from the mean mass using Eqs. (9) and (10) for the lowest, intermediate and highest redshift bins. The actual mean metallicity and SFR for these samples are shown with crosses (see Sect. 4.3).

and shape is not directly comparable to the GAMA MMR presented here. Nevertheless, the similarity between the SDSS and GAMA MMRs is encouraging to see given the inherently different apparent magnitude ranges probed by the two surveys.
Indeed, although GAMA covers a smaller volume than SDSS, it is probing to fainter magnitudes, and a galaxy of a given mass will on average be detected at higher redshift in GAMA than in SDSS. This consideration suggests that any evolutionary 
effects over this redshift range are likely to be small. A companion paper (Lara-López et al., in prep.) compares the SDSS and GAMA surveys directly and self-consistently.

As is common, we fit a second order polynomial to the MMR in GAMA and obtain

$$
\begin{aligned}
\log (\mathrm{O} / \mathrm{H})+12= & (-2.2 \pm 0.3)+(1.95 \pm 0.07) \times \log \left(M_{*}\right) \\
& -(0.085 \pm 0.003) \times \log \left(M_{*}\right)^{2}
\end{aligned}
$$

with an rms scatter of $\sigma=0.12 \mathrm{dex}$, similar to that found for SDSS (e.g., Tremonti et al. 2004; Kewley \& Ellison 2008). Equation (9) is valid for $8.7 \leq \log \left(M_{*}\left(M_{\odot}\right)\right) \leq 12.5$ for our fiducial selection criteria and the PP04 metallicity calibration. We emphasize here that the MMR fit in this fashion arises from the contribution of galaxies at all redshifts probed in GAMA $(0.061 \leq z \lesssim 0.4)$. As a result, galaxies at low (high) redshift generally contribute to the low (high) mass end of the MMR, and hence possible evolutionary processes are likely to be masked.

\subsection{Mass-SFR relation}

The upper right panel of Fig. 6 shows the relationship between stellar mass and SFR in GAMA for our main sample as well as the 14 volume limited redshift samples. While the relationship is difficult to detect in the individual volume limited samples due to the small stellar mass range probed in each one, we find a positive correlation between $\log \left(M_{*}\right)$ and SFR in the main sample, spanning the full redshift range probed. We use a bisector fit for galaxies in the main sample with reliable stellar mass and SFR measurements after applying a $2 \sigma$ clipping. We obtain:

$\log (S F R)=(1.304 \pm 0.008) \times\left(\log \left(M_{*}\right)\right)-(12.98 \pm 0.08)(10)$

with a normal rms scatter of $\sigma \sim 0.5$ dex. As with the MMR, we find that all the volume-limited samples are consistent with the global fit, and the overall trend is consistent with that found in the SDSS, although the global fit is still affected by the Malmquist bias.

\subsection{Metallicity-SFR relation}

The lower panel in Fig. 6 shows the relationship between metallicity and SFR for galaxies in GAMA. We find only a weak relation between the two in only some of our volume-limited redshift samples. The main sample itself doesn't exhibit any correlation. In order to better understand the effect of the MMR and mass-SFR relationships measured in Sects. 4.1 and 4.2 on the metallicity-SFR distribution, we perform the following exercise. We compute the mean stellar mass in each volume-limited sample and use Eqs. (9) and (10) to infer a corresponding metallicity and SFR, respectively. These are shown for the lowest, intermediate and highest redshift bins as open circles in the lower panel of Fig. 6. The actual mean metallicity and SFR for these samples are shown with crosses. While Eqs. (9) and (10) provide a consistent estimate for the position of the data at the intermediate redshift samples, they seem to over- (under-)predict the metallicity at low (high) redshift by $\sim 0.05$ dex. This result is likely to arise as a consequence of the fits to the whole sample being dominated by the intermediate redshift samples where the data density is greatest. The implication, though, is that at the higherredshift end the observed metallicity is slightly lower than would be consistent with the extrapolation from the intermediate redshift objects. Conversely, at the lower-redshift end, the observed metallicity (and SFR) is slightly higher $(\sim 0.2 \mathrm{dex})$. This may be suggestive of evolutionary effects, even over this redshift range (see also Lara-López et al., in prep.).

\section{Summary and conclusions}

We have presented the dependence of the mass-metallicity relationship on a variety of selection criteria, metallicity calibrations and SFR. As in previous studies, we see the strong massmetallicity relationship for the GAMA samples. The shape and position of the mass-metallicity relationship can vary significantly depending on the metallicity calibration and selection used. In particular, we find that selecting on the [OIII] $\lambda 5007$ line most affects the position and shape of the mass-metallicity relationship, while varying survey depth does not. Alternatively, for monotonic metallicity calibrations such as that of Pettini \& Pagel (2004), one can also select on the metallicity uncertainties itself without biasing the sample. Given these, caution must be used when comparing the mass-metallicity relationship obtained using different metallicity calibrations and/or sample selection criteria.

The mass-metallicity relationship in GAMA is in reasonable agreement with that found in SDSS despite the very different stellar mass range probed at any given redshift. Notwithstanding the cautions illustrated in this work, this result implies that evolutionary effects out to $z<0.35$ are likely to be small. Such small effects reinforce the importance of caution in selecting samples with which to estimate their measurement.

Using volume-limited redshift samples, we find tentative evidence that galaxies at lower redshift may indeed have measurably higher metallicities than those at higher redshift, even over the redshift range probed within GAMA. This suggests that there could be detectable evolution of the MMR on the order of a few tenths of a dex in metallicity spanning $0.06<z<0.35$.

In a companion paper (Lara-López et al., in prep.), we investigate the joint SDSS + GAMA mass-metallicity relationship, its evolution and the joint 3D distribution in metallicity- $\log \left(M_{*}\right)$ SFR space self-consistently and in detail.

Finally, while the use of fibre-fed spectrographs has enabled large redshift surveys up to now, all are essentially limited by aperture biases. Indeed, it is imperative to test the basic assumptions generally used in similar work that 1) star formation follows the distribution of stellar light and 2) models of single HII regions can be applied to spectra of entire galaxies. These assumptions can now be tested using available integral field unit spectrographs by observing galaxies at low redshift. Moreover, the Sydney-AAO Multi-object Integral field spectrograph with its hexabundle technology Croom et al. (2012) will allow large spatially resolved spectroscopic galaxy surveys, enabling us to test these important assumptions and eliminating aperture biases.

Acknowledgements. C.F. thanks the Australian Astronomical Observatory for financial support in the form of a graduate top-up scholarship. This work was co-funded under the Marie Curie Actions of the European Commission (FP7-COFUND). GAMA is a joint European-Australasian project based around a spectroscopic campaign using the Anglo-Australian Telescope. The GAMA input catalogue is based on data taken from the Sloan Digital Sky Survey and the UKIRT Infrared Deep Sky Survey. Complementary imaging of the GAMA regions is being obtained by a number of independent survey programs including GALEX MIS, VST KIDS, VISTA VIKING, WISE, Herschel-ATLAS, GMRT and ASKAP providing UV to radio coverage. GAMA is funded by the STFC (UK), the ARC (Australia), the AAO, and the participating institutions. The GAMA website is http://www.gama-survey.org/.

\section{References}

Abraham, R. G., Glazebrook, K., McCarthy, P. J., et al. 2005, in Starbursts: From 30 Doradus to Lyman Break Galaxies, eds. R. de Grijs, \& R. M. González Delgado, Astrophysics and Space Science Library, 329, 195

Baldwin, J. A., Phillips, M. M., \& Terlevich, R. 1981, PASP, 93, 5

Beifiori, A., Maraston, C., Thomas, D., \& Johansson, J. 2011, A\&A, 531, A109 
Bresolin, F., Gieren, W., Kudritzki, R.-P., et al. 2009, ApJ, 700, 309

Brinchmann, J., Charlot, S., Heckman, T. M., et al. 2004

[arXiv: astro-ph/0406220]

Bruzual, G., \& Charlot, S. 2003, MNRAS, 344, 1000

Calzetti, D. 2001, PASP, 113, 1449

Cappellari, M., \& Emsellem, E. 2004, PASP, 116, 138

Cardelli, J. A., Clayton, G. C., \& Mathis, J. S. 1989, ApJ, 345, 245

Carollo, C. M., \& Lilly, S. J. 2001, ApJ, 548, L153

Cowie, L. L., Songaila, A., Hu, E. M., \& Cohen, J. G. 1996, AJ, 112, 839

Croom, S., Saunders, W., \& Heald, R. 2004, Anglo-Australian Observatory Epping Newsletter, 106, 12

Croom, S. M., Lawrence, J. S., Bland-Hawthorn, J., et al. 2012, MNRAS, 421, 872

Driver, S. P., Hill, D. T., Kelvin, L. S., et al. 2011, MNRAS, 413, 971

Ellison, S. L., Patton, D. R., Simard, L., \& McConnachie, A. W. 2008, ApJ, 672, L107

Erb, D. K., Shapley, A. E., Pettini, M., et al. 2006, ApJ, 644, 813

Ferreras, I., La Barbera, F., de Carvalho, R. R., et al. 2012, MNRAS, in press [arXiv: 1206.1594]

Finlator, K., \& Davé, R. 2008, MNRAS, 385, 2181

Gallazzi, A., Charlot, S., Brinchmann, J., White, S. D. M., \& Tremonti, C. A. 2005, MNRAS, 362, 41

Garnett, D. R. 2002, ApJ, 581, 1019

Gunawardhana, M. L. P., Hopkins, A. M., Sharp, R. G., et al. 2011, MNRAS, 415,1647

Hopkins, A. M., Miller, C. J., Nichol, R. C., et al. 2003, ApJ, 599, 971

Kauffmann, G., Heckman, T. M., Tremonti, C., et al. 2003, MNRAS, 346, 1055

Kennicutt, Jr., R. C. 1998, ApJ, 498, 541

Kewley, L. J., \& Dopita, M. A. 2002, ApJS, 142, 35

Kewley, L. J., \& Ellison, S. L. 2008, ApJ, 681, 1183

Kewley, L. J., Dopita, M. A., Sutherland, R. S., Heisler, C. A., \& Trevena, J. 2001, ApJ, 556, 121

Kobayashi, C., Springel, V., \& White, S. D. M. 2007, MNRAS, 376, 1465

Kobulnicky, H. A., \& Kewley, L. J. 2004, ApJ, 617, 240

Kodama, T., Yamada, T., Akiyama, M., et al. 2004, MNRAS, 350, 1005

Köppen, J., Weidner, C., \& Kroupa, P. 2007, MNRAS, 375, 673

Lara-López, M. A., Bongiovanni, A., Cepa, J., et al. 2010a, A\&A, 519, A31

Lara-López, M. A., Cepa, J., Bongiovanni, A., et al. 2010b, A\&A, 521, L53

Lara-López, M. A., Cepa, J., Bongiovanni, A., et al. 2009a, A\&A, 493, L5

Lara-López, M. A., Cepa, J., Bongiovanni, A., et al. 2009b, A\&A, 505, 529

Lara-Lopez, M. A., Lopez-Sanchez, A. R., \& Hopkins, A. M. 2012, ApJ, submitted [arXiv: 1207.0950]

Larson, R. B. 1974, MNRAS, 169, 229

Laskar, T., Berger, E., \& Chary, R.-R. 2011, ApJ, 739, 1

Lequeux, J., Peimbert, M., Rayo, J. F., Serrano, A., \& Torres-Peimbert, S. 1979, A\&A, 80, 155

López-Sánchez, Á. R., \& Esteban, C. 2010, A\&A, 517, A85

Lopez-Sanchez, A. R., Dopita, M. A., Kewley, L. J., et al. 2012, MNRAS, in press [arXiv: 1203.5021$]$

Maiolino, R., Nagao, T., Grazian, A., et al. 2008, A\&A, 488, 463

Mannucci, F., Cresci, G., Maiolino, R., et al. 2009, MNRAS, 398, 1915

Mannucci, F., Cresci, G., Maiolino, R., Marconi, A., \& Gnerucci, A. 2010, MNRAS, 408, 2115

Maraston, C. 2005, MNRAS, 362, 799

Maraston, C., \& Strömbäck, G. 2011, MNRAS, 418, 2785

McGaugh, S. S. 1991, ApJ, 380, 140

Mendel, J. T., Proctor, R. N., Rasmussen, J., Brough, S., \& Forbes, D. A. 2009, MNRAS, 396, 2103

Morelli, L., Calvi, V., Cardullo, A., et al. 2012, A\&A, 544, A74

Mouhcine, M., Bamford, S. P., Aragón-Salamanca, A., Nakamura, O., \& Milvang-Jensen, B. 2006, MNRAS, 369, 891

Moustakas, J., Kennicutt, Jr., R. C., Tremonti, C. A., et al. 2010, ApJS, 190, 233

Moustakas, J., Zaritsky, D., Brown, M., et al. 2011, ApJ, submitted [arXiv: 1112.3300]

Pettini, M., \& Pagel, B. E. J. 2004, MNRAS, 348, L59

Pilyugin, L. S., \& Thuan, T. X. 2005, ApJ, 631, 231

Rodrigues, M., Hammer, F., Flores, H., et al. 2008, A\&A, 492, 371

Rodrigues, M., Puech, M., Hammer, F., Rothberg, B., \& Flores, H. 2012, MNRAS, 421, 2888

Salpeter, E. E. 1955, ApJ, 121, 161
Sánchez-Blázquez, P., Peletier, R. F., Jiménez-Vicente, J., et al. 2006, MNRAS, 371,703

Sarzi, M., Falcón-Barroso, J., Davies, R. L., et al. 2006, MNRAS, 366, 1151

Savaglio, S., Glazebrook, K., Le Borgne, D., et al. 2005, ApJ, 635, 260

Sharp, R., \& Birchall, M. N. 2010, PASA, 27, 91

Sharp, R., \& Parkinson, H. 2010, MNRAS, 408, 2495

Spitoni, E., Calura, F., Matteucci, F., \& Recchi, S. 2010, A\&A, 514, A73

Taylor, E. N., Hopkins, A. M., Baldry, I. K., et al. 2011, MNRAS, 418, 1587

Thomas, D., Maraston, C., Schawinski, K., Sarzi, M., \& Silk, J. 2010, MNRAS, 404, 1775

Tremonti, C. A., Heckman, T. M., Kauffmann, G., et al. 2004, ApJ, 613, 898

Wilkins, S. M., Hopkins, A. M., Trentham, N., \& Tojeiro, R. 2008a, MNRAS, 391,363

Wilkins, S. M., Trentham, N., \& Hopkins, A. M. 2008b, MNRAS, 385, 687

Yates, R. M., Kauffmann, G., \& Guo, Q. 2012, MNRAS, 422, 215

Zahid, H. J., Kewley, L. J., \& Bresolin, F. 2011, ApJ, 730, 137

Zaritsky, D., Kennicutt, Jr., R. C., \& Huchra, J. P. 1994, ApJ, 420, 87

1 European Southern Observatory, Alonso de Cordova 3107, Vitacura, Santiago, Chile e-mail: cfoster@eso.org

2 Centre for Astrophysics \& Supercomputing, Swinburne University, Hawthorn, VIC 3122, Australia

3 Australian Astronomical Observatory, PO Box 296, Epping, NSW 1710, Australia

4 Sydney Institute for Astronomy, School of Physics, University of Sydney, NSW 2006, Australia

5 The Australian National University, Mount Stromlo Observatory, Cotter Road, Weston Creek, ACT, 2611, Australia

6 Institute of Cosmology and Gravitation (ICG), University of Portsmouth, Dennis Sciama Building, Burnaby Road, Portsmouth PO1 3FX, UK

7 International Centre for Radio Astronomy Research, The University of Western Australia, 35 Stirling Highway, Crawley, WA 6009, Australia

8 School of Physics \& Astronomy, University of St Andrews, North Haugh, St Andrews, KY16 9SS, UK

9 Astrophysics Research Institute, Liverpool John Moores University, Twelve Quays House, Egerton Wharf, Birkenhead, CH41 1LD, UK

10 Centre for Astronomy and Particle Theory, University of Nottingham, University Park, Nottingham NG7 2RD, UK

11 European Southern Observatory, Karl-Schwarzschild-Str. 2, 85748 Garching, Germany

12 Astronomy Centre, University of Sussex, Falmer, Brighton BN1 9QH, UK

13 Institute for Computational Cosmology, Department of Physics, Durham University, South Road, Durham DH1 3LE, UK

14 Institute for Astronomy, University of Edinburgh, Royal Observatory, Blackford Hill, Edinburgh EH9 3HJ, UK

15 Department of Physics, Swiss Federal Institute of Technology (ETH-Zürich), 8093 Zürich, Switzerland

16 School of Physics, Monash University, Clayton, 3800 Victoria, Australia

17 Leiden University, P.O. Box 9500, 2300 RA Leiden, The Netherlands

18 Jeremiah Horrocks Institute, University of Central Lancashire, Preston PR1 2HE, UK

19 Department of Physics and Astronomy, Macquarie Uiversity, NSW 2109, Australia

20 Astronomy Unit, Queen Mary University London, Mile End Rd, London E1 4NS, UK

21 South-East Physics Network (SEPnet)

22 Max Planck Institute for Nuclear Physics (MPIK), Saupfercheckweg 1, 69117 Heidelberg, Germany 